\title{
The Construction of Trust in Public Governance : Based on the Perspective of Cooperative Governance
}

\author{
Jian Min ${ }^{1}$ Chen Lian' \\ ${ }^{1,2}$ School of Politics and Public Affairs,Southwest University of Political Science and \\ Law,Chongqing,P.R.China,401120 \\ (E-mail:jianmin1998@126.com,673551933@qq.com)
}

\begin{abstract}
In the risky society, social contradictions and conflicts have been showing up endlessly. "Trust" as an important social capital is facing with huge damage. The contemporary social cross boundary risk characteristics ask for more governance and make access to a relationship of cooperation. This paper attempts to take the method of comparative studies and literature studies, and within the combination of relevant empirical case, mainly to study the origin and necessity of rebuilding trust in the cooperative governance, and then analyze the great promoting function of NGOs into full play in cooperative management with the cooperative trust, lastly put forward the strategy of rebuild trust relationship for the phenomena of voluntary failure, insufficient revenue sharing, uneven risk-sharing.
\end{abstract}

Keywords: Cooperative Governance, Reconstruction of Trust, NGO, Trust Dilemma, Cooperative Trust

\section{Introduction}

The complexity of the contemporary public affairs and the frequentness of public crisis change the management mode from the traditional single government governance to modern diverse cooperative governance. And in the cooperative governance, the operation and development of non-governmental organizations are facing with great challenges of the reconstruction of trust.

A number of basic theories can help us understand the trust building in cooperative management. Different scholars adopt different ways to explain the theories, including Salamon's Failure theory, Pennings' Resource dependence theory, Zhang Kangzhi's Governance theory, Robert. Putnam's Social capital theory. These theories constitute the theoretical background and framework structure for solving problem deeply, but these theories have its own limitations for strategy analysis. To understand well about the background of the reconstruction of trust in the cooperative governance, we can begin with three aspects. Firstly, for NGO, the theory of management has become the basic theoretical background of promoting the reconstruction of trust. As a new form of governance, the cooperative governance is a management mode which is based on the friendly sentiments of interactive relationship. 
Secondly, the spring up and the booming development of NGO become the basic organization premise to promote the reconstruction of trust in the cooperative governance. Thirdly, the phenomenon and damage of trust deficiency has been the direct cause of the reconstruction of trust. Recently, the trust crisis triggered by the risk management of various aspects increases continually in number and its influence is spinning on a daily basis, such as "peek-a-boo events" in Yunnan Province, "Bao'an events" in Shenzhen Province, 5.12 earthquakes Sichuan Province, the pollution of Songhua River and so on.. It means that the reconstruction of the trust relationship in the cooperative governance is urgent.

\section{The importance of trust in cooperative governance}

\subsection{On the way to cooperative governance}

Risk society makes the way to cooperative governance. A "risk society" is based on the solution of the problems--how to avoid, minimize or guide the risk and harm which is part of the modernization and produced systematically. [1] Since the 1980s, globalization has not only been the economic globalization, financial globalization, cultural globalization and technological globalization, but also been a kind of risk globalization. We are entering a "risk society" presupposed by Baker. The order of risk society is not the type of social estate or the vertical one, but the type of network and plane extended. As risks never look over anybody, this is "the equality activists". And across-the-border characteristics of risk ask for the appearance of more subjects and the contribution to the cooperation relations. Fortunately, cooperative governance is the effective model which adapts to risk society.

Cooperative governance has broken the "center-edged" structure, changing from the "single- centered" control to "several-centered" cooperation. Facing with the environment which is full of complexity, dynamic and diversity, the government is no longer the only "original discourse". What we really need is the common management with the "discourse" produced by the interaction of various social strengths. [2] First of all, to realize the "self-emancipation" of the government, we should gradually promote its right decentralization to social, and establish the "small government of management"," strong government of service" by the transformation of government functions. Thereafter, we should do our best to actively cultivate, develop and regulate the management of social organization. In this way, we might try our best to create the "great common-managed social ", "the harmony fantastic society".

\subsection{Analysis of the importance of the trust relationship in the cooperative governance}

According to the basic historical form of agricultural society, industrial society and the industrial society and the communication and human history form of acquaintances society and strange society, some scholars divide the trust into the customary trust, the compact trust and the cooperative trust. [3] In this article, we choose the concept of generalized cooperation - the cooperation between NGO and government to achieve a common goal of public service, the functions which strengthen the embedding cooperation and improve the effectiveness of work and revenue sharing. So the trust relationship studied in this paper is the cooperative trust which is the precondition 
of the cooperative governance and the product of successful cooperation management.

Trust is the lubricant of cooperation and it is also the fundamental of the cooperation relationship. At the same time, distrust also destroys the cooperation. If fully distrust, the cooperation of free actors will fail. Therefore, trust mechanism is the foundation of the cooperative governance of public resources. Whether they could get rid of the collective action problem and realize the effective management of public resources depends on the trust relationships and the degree of them among various kinds of main participated subjects.

\section{Trust relationships in collaborative governance contributes to promote the NGO functions}

To some extends, government expects NGO to participate in public affairs by utilizing folk resources, it has even depended on the resources possessed by NGO.[4]This article below will explain how the collaborative governance would promote NGO functions.

\subsection{Trust relationship contributes to promote the NGO's function of infrastructure service and make up the vacuum in governments and markets}

NGO gives full play to the function of making up the deficiency in governments and markets in the process of social services and management, which is based on the alternative service function that governments and markets exerts on the trust relationship, it is a remedy after "government failure" and "market failure". On one hand, trust relationship help further the communication between governments and society, promoting its function of meeting social diversity needs in the process. On the other hand, the nonprofit and voluntary characteristics of NGO deepen the trust from governments and society, which help NGO optimize qualities of social management better.

\subsection{Trust relationship helps NGO lower the} cost of participating in collaborative management and enhance the utility of supplying public goods

Building up trust mechanism would make the communication between cooperation participants more efficient, thus reducing the moral risks caused by information asymmetry and opportunism. And it would also give expectations between collaborators. If they develop mutual trust, the mechanism of self-supervision and restriction will be built. Therefore, it is unnecessary to carry out their oral and formal contract by outside power such as judicial departments, which could result in lowering the price of governments' supervision and executions, releasing more resources for NGO to use in public management and improving the efficiency of collaborative management for public resources.

\subsection{NGO could better relieve social tension} and balance social interest with the help of

\section{trust relationship}

The non-profit feature of NGO highlights the neutrality when dealing with individual interest and the mutual interest with organization. NGO acted as a medium for communication between governments and public. Based on the stable trust relationship, public could supervise exertion of public power by means of NGO to achieve fair distribution of public interests, which would not only benefit those who have the same preference as the governments, those who represent advanced social productivities and 
most people's interest, but also concern the interests of leftover children and vulnerable groups who live in the rural and backward area to achieve "fair distribution" by balancing public interest, individual interest and mutual interest with organization.

\subsection{Trust relationship contributes to the political function of NGO and help to build up the harmonious society}

In the process of cooperation management, the high level of trust could encourage network participants to act flexibly and promptly and follow the contract, reducing the unnecessary conflicts and negotiations and time costs of dealing with disputes. Moreover, trust relationship could reflect the needs of environmental changes promptly.[5] For most people who are indifferent to political participation, the enthusiastic service of NGO may better stimulate their desires of participating in politics and its' way of communication is also easily accepted by the public, by which NGO could build up and perfect communication channel and negotiation mechanism of public interest. Governments and public need such a buffered and progressive process as forming the institutionalized interest expressing system to lead public express their needs rationally and legally.

\section{Trust crises in current cooperative management}

Although trust mechanism plays a very important role in cooperative management, there still exist elements of trust crisis, which inevitably impede the realization of cooperative management.

\subsection{The factors of trust crisis in cooperative management}

4.1.1. Changes in ways of cooperation causes trust crisis

Since reform and opening-up, our nation has gone into the period of transition, transforming from the closed and traditional social structure to modern social structure, from highly centralized planned economy to free and open socialist market economy, from single central control pattern to multiply central controls. The cooperative principle, idea and goal have all changed correspondingly. The Innate interest structure in society and trust relationship that have already been built up during the transformation period have formed an separate and close relationship in current social structure and management pattern. In this case, trust relationship of governments, society and NGO would no longer be the traditional trust relationship and contractual trust relationship. While the newly-built trust relationship in cooperation would surely obtain the elements of previous trust relationship. It would definitely bring about trust crisis when basis and ways generating trust between governments, NGO and society changes.

4.1.2. The restriction of trust rule speeds up the pace of generating trust crisis

With the development of economic globalization, cultural globalization, technology globalization, risks globalization, nations have formed crisscross and complicated international web relationship system and formed mutual conditioned and influenced chain restriction. For example, the financial crisis in 2008 affected several nations. However, the restriction power of trust rule inside a nation weakens gradually. The invisible restriction of trust rule would play a part when social members have a high degree of dependence on mutual assistance in 
economy, allocation of risks and share of interest. However, china now is in the transition period and has gradually transformed to the "anonymous society", with the distinctive features as mobility, openness, low-frequency contact. The restriction power caused by breaking the rules has also weakened in the cooperative management. The instability and openness of invisible conditioned relationship between different social powers impede the progress in cooperative management, which is also known as the "catalyst" brought by trust crisis.

\subsubsection{Lack of official system deepen trust crisis}

Setting up an official system is based on formal regulations, institution and law. When one subject in the cooperative relationship breaks the rules of institution, it may suffer a loss and be punished. When institution operates well, cooperative subjects would naturally make the system internalized and form a stable institutionalized relationship. However, the fact that some previous restriction power of institution has gradually weaken and newly established system could not be built up quickly caused some vacant space and make some discreditable behaviors happed in cooperative relationship between governments, NGO, and markets fail to receive penalty. Trust relationship between NGO and governments would not get institutionalized guarantee, which deepen the trust crisis. Setting up wholesome institutionalized guarantee system is of great value to build up and maintain the trust relationship. Or, lack of institution would deepen trust crisis and bring about trust crisis in many other aspects.

\subsection{The manifestation of trust crisis in cooperative management}

There are various shows of trust crisis, which mainly show in the following aspects:

\subsubsection{Voluntary failure}

Salamon, the first person who puts forward the voluntary failure theory, contends that non-governmental organization exist some problems such as lack of charity, paternalism, amateur, limitations of service objects. [6]Although non-governmental organizations would complement public good supply, which is the third option after "government failure" and "voluntary failure", they can not promote charities and programs for public welfare all by themselves. This limitation is known as "voluntary failure". The current voluntary failure analysis can not live without the analysis of trust relationship between governments, NGO and markets. Voluntary failure is mainly reflected in the "governmental" and "mercerization" of NGO. [7]On one hand, after reform and opening up, it formed the resources from markets and aboard. NGO is faced with opportunities and challenges, including how to use the resources properly while guarantee the non-governmental features. On the other hand, in order to function well in the operation of charities, NGO must rely on the market operation mechanism to open up source of wealth, compete with enterprise and engage in some profitable activities. This is the marketization of NGO. In order to broaden the diversities of funds sources and reduce the dependence on organizations, many NGOs take part in more and more profitable activities. When they pursue profits just as many commercial organizations, the non-governmental features prove to be a thoughtful question.

\subsubsection{Insufficient gain sharing}

Income level made in the process of sharing 
cooperative management is always the lowest because of the charitable and non-profit features. From governments' point of view, they have great possibilities to confer some advantages and ignore the material and policy support required by its' own development. From the market view, it is free to enter or quit. Moreover, based on the "rational economic" assumption, when the maximum power and goal is to seek profits, markets would rather risk destroying the collaborative relationship to occupy the gain sharing of NGO for more gain sharing. What' $s$ more, collaboration in any kind or scale would not always make profit, only when governments and NGO achieve cooperation of reciprocal benefits and shared value, would they collaborate well with each other and share profit. The pursuit of reaching maximum utilities can not live without the restriction of collaborative scale. Only when cost of production and transaction reach the balance, can we achieve the best cooperation scale and optimal cooperative profits, thus achieving the optimal interest sharing structure. [8]

\subsubsection{The uneven distribution of risks}

The uneven distribution between governments, NGO and markets is an important demonstration of trust crisis in the process of cooperation. In the risk society, the probabilities of risks happened to every organization and person should be average. The only difference is the degree of risk sharing that every organization should take in cooperation. On one hand, under the condition of market economy, business organizations have options and flexibilities to some degree when entering and quitting the cooperation relationships. The lack of punishments to the action of destroying cooperative relationship in market rules can not act as a deterrent to the action of escaping the risks. Governments in the cooperative relationship often act as a protagonist in making institutions, regulations and even law. They consider less of the loss in NGO itself. On the other hand, reconsideration and litigation are based on the opposition while cooperative relationship is based on the partnership which has the same interests. Using previous ways is detrimental to cooperation and ensuring interests of NGO and social enterprises. What's more, reconsideration and litigation are remedy mechanism and ways to resolve the problems afterwards, which go against the starting point of responding the public quickly. This kind of lag solution will make the risks last longer and more risks would be shared by NGO and governments, enlarging and deepening trust crisis.

\section{The strategy of reconstructing trust in cooperative governance}

Some scholars think that there are three ways of producing trust. Firstly, trust is based on system. It depends on the formal system arrangement as well as intermediate mechanism. Secondly, trust is based on progress. Trust is resulted from the expectations of the past and the future. Thirdly, trust is based on social culture. The key of successful cooperation between actors with different cultural backgrounds is seeking common ground while reserving differences.The following paragraphs will discuss the reconstructive strategy of trust relationship from the points of government, NGO and society.

\subsection{It is important to perfect the system base of reconstructing trust in cooperative governance}


On one hand, policy system of supporting NGO should be established. The system is made up of three main aspects. The first aspect is to promote the government departments to transfer some micro-level work, social service work and service function to NGO. The second aspect is that government should purchase the service of NGO so as to develop the function of NGO. The last aspect is to cultivate the talents of NGO. Promoting to perfect the preferential tax policy is also included. The construction of trust requires breaking the situation of government monopoly rights and establishing the free and equal discourse mechanism so as to meet the development needs of NGO. All the participants can express their political suggestions and ideas and they can explain them.

On the other hand, it is important to ensure the orderly and healthy development of NGO by laws. The government should fill the legal blind spot as soon as possible as well as improve the NGO legal and policy environment. The government also needs to strengthen supervision and management and to standardize the behaviors of NGO. At the same time, the government is supposed to prevent the administrative departments through legislation to strengthen the power of government departments so as to change the used situation that too much importance has been attached to the registration and administrative management while the government ignored the management and management according to the law. It means that the government will provide basis and support from the law level for the healthy development of NGO and help the NGO to improve itself. Measures like these will change the double management mechanism implemented by the country before.

\subsection{It's essential to enhance the organizational strength of reconstructing trust in cooperative governance}

To begin with, the NGO should strengthen its independence and reduce the dependence on government. The NGO can enhance its ability by establishing the constructions of inspection, evaluation, information disclosure mechanism while strengthen the construction of resource conformity, commonweal and self-discipline mechanism. Strengthening the construction of public welfare and self-discipline mechanism is the effective way of sustaining the nonprofit and voluntary function of NGO. For lacking essential social public confidence, NGO has difficulties in raising funds form society. As a result, the NGO has to depend on the government funding, reducing NGO's independence and its function of supervising and restricting the government power. Only when the NGO improve its independence can it cooperate with the government.

Second of all, NGO should introduce market mechanism as to strengthen the ability to provide social service and develop itself. The non-profit of NGO leads to the fact that the capital of NGO mostly comes from the governmental support and social fund. However, both ways will reduce the independence of NGO and have negative effects on the non-government organization's development and function. As a result, the NGO need to widen financing source channel and find more funds to develop by it. The introduction of market competition mechanism makes it possible that NGO can follow the mode of the enterprise to seek development. Thus the NGO can enhance its competitiveness. But it is a great challenge for 
commonweal maintain. Therefore, the NGO shall also establish evaluation system to enhance the competitiveness of $\mathrm{NGO}$ and ensure its non-profit attribute.

\subsection{It's important to enhance the social capital of reconstructing trust in cooperative governance}

From the point of society, we should raise the level of social and economic development and education, learn and excavate optimal traditional culture. We also need to take active parts in developing society and promote the increase of social capital such as "trust" Putnam has conducted a survey about the contemporary American social capital development trend and has analyzed the various factors which make the fall of social capital of United States. And then he put forward his idea of maintaining the citizen participation and civic trust.[9].Social capital means the characteristics of social organizations such as trust, rules and the Internet. They can improve social efficiency through pushing coordination and action. Thus, trust is an essential part of social capital and is a social lubricant. The development of civic society is closely associated with the trust social capital. It unites the participants of public resource network governance through the horizontal link of coordination. With the development of civic society, the network of the horizontal relationship between society and politics has been improved. Correspondingly, the degree of equal treatment, mutual respect and mutual trust between each other through the network has been improved. And the users of the network are willing to abide by the laws and regulations and social ethics. Accordingly, we will achieve social trust and then to promote to realize the goal of public resource network management.

\section{Conclusions}

This paper attempts to take the method of comparative studies and literature studies, and within the combination of relevant empirical case, mainly to study the origin and necessity of rebuilding trust in the cooperative governance, and then analyze the great promoting function of NGOs into full play in cooperative management with the cooperative trust, lastly put forward the strategy of rebuild trust relationship for the phenomena of voluntary failure, insufficient revenue sharing, uneven risk-sharing. This paper is not only to fully affirm the trust relationship effectively promote cooperation management process, but also to soberly deny its lagging development and many crisis emerging in the social process of governance; meanwhile, it is the urgent need of solving some outstanding problems in current social modernization process and building a "harmonious society", and for the considering of scientific and standardization in social management under the background of the current rapid economic development.

\section{References}

[1][Germany] Beck. The society of risks [M].Translated by Bowen He, Yilin Press, 2004. (In Chinese)

[2]Zhengbo Peng. The interpretation of public administration from the view of post modern [J].copy data from Renmin University of China. Public administration, 2005, (4). (In Chinese)

[3] Kangzhi Zhang. Talking about trust in history-three historic types of trust [J].Social Science Research, 2005, (1). (In Chinese) 
[4] Shiwei Chen. The research about the trust relationship between government and NGO: From the view of the cooperative governance mode [J].Jiangxi Social Science, 2008,(11).(In Chinese)

[5]Jones G.R,George J.M.The Experience and Evolution of Trust: Implications for Cooperation and Teamwork[J].Academy of Management Review,1998,23(3).

[6] Leicester.Salamon. Global civil society-From the view of nonprofit sector [M].Translated by Xijing Jia,Yu Wei, etc. Social Science Literature Press, 2002.(In Chinese)
[7] Ming Wang,Lei Tong.The views and future of Qinghua's research about NGO [J]. Chinese administrative management, 2003,(3).(In Chinese)

[8]Heng Xi,Xiaokang Lei. The system base of Harmonious Society and the basic task of public management [J].Journal of Northwestern University,2008,(3).(In Chinese)

[9][U.S.]Robert.Putnam.Bowling Alone-The Collapse and Revival of American Community[M].Translated by Liu Bo,Naijuan Zhu,etc.Beijing University Press,2011.(In Chinese) 